http://jmscr.igmpublication.org/home/ ISSN (e)-2347-176x ISSN (p) 2455-0450 crossref DOI: https://dx.doi.org/10.18535/jmscr/v9i6.13

\title{
Functional Outcome of Cemented Bipolar Hemiarthroplasty For Unstable Intertrochanteric Femur Fracture in Elderly Osteoporotic Patients
}

\author{
Authors \\ Dr Aswin Thankachan. $\mathbf{V}^{1}$, Dr Jagajeev. JR ${ }^{2 *}$, Dr Sabarisree $\mathbf{M}^{3}$ \\ ${ }^{1}$ Postgraduate Student in MS Orthopaedics, Government Medical College, Thiruvananthapuram \\ ${ }^{2,3}$ Additional Professor, Department of Orthopaedics, Government Medical College, Thiruvananthapuram \\ *Corresponding Author \\ Dr Jagajeev. JR \\ Additional Professor, Department of Orthopaedics, Government Medical College, Thiruvananthapuram
}

\begin{abstract}
Introduction: Hip fractures in elderly are frequent and their number is increasing fast. Intertrochanteric hip fractures account for approximately half of the hip fractures in elderly; out of this more than 50\% fractures are unstable.

In osteoporotic elderly patients, primary stabilization with dynamic hip screws has high failure rates due to implant failure, cut-out of head and penetration into hip. Treatment with cemented bipolar hemiarthoplasty in such patients lessen the duration of recumbency, early rehabilitation the patient, decreases the pulmonary post-operative complications and return of these subjects to the pre-injury level of activity more quickly.

Methodology: This is a longitudinal study, in which 24 patients with age $>70$ years who had unstable intertrochanteric fracture with no major associated injury who came to orthopedics department, Thiruvananthapuram Medical College, were treated with cemented bipolar hemiarthroplasty.

Result: Patients who underwent cemented bipolar hemiarthroplasty, at 2 weeks, 6 weeks, 3 moths had better Harris hip score than who underwent DHS and they were statistically significant $(p<0.001)$. They were more pain free and had greater range of hip movements. They could be mobilized very early compared with the other group and were able to presume their daily activities early than other group. Harris hip score at final follow up at 1 year in both groups were comparable and no significant difference couldn't be found out statistically.

Conclusion: After analyzing functional results we conclude that this method is well tolerated by aged patients. Early ambulation is possible thus lessening the chance of developing pulmonary complications and bed sores and a resurgery is less frequently required. This also improves their quality of life and the ability to go back to their original functional status much earlier than DHS.

Keywords: Cemented bipolar hemiarthroplasty, DHS, functional; outcome early mobilization.
\end{abstract}

\section{Introduction}

Hip fractures in elderly are frequent, and their number is increasing fast. Intertrochanteric hip fractures account for approximately half of the hip fractures in the elderly; out of this more than $50 \%$ fractures are unstable.

The factors most significant for instability and fixation failure are (1)Loss of posteromedial 
support (2)Severe comminution (3)Sub trochanteric extension of the fracture (4)Reverse oblique fracture (5)Shattered lateral wall (6)Extension into femoral neck area and (7)Poor bone quality.

Unstable patterns occur more commonly with increased age and with low bone mineral density. The results of unstable fractures are less reliable and have a high rate of failure (8\%-25\%). Major problems involved in these fractures are (1)Association with substantial morbidity and mortality due to prolonged immobilization (2)Malunion (3)Great financial burden to the family (4)Associated medical problems like diabetes and hypertension.

In osteoporotic elderly patients, primary stabilization with dynamic hip screws/sliding screws is not always successful. In order to rehabilitate the patient and lessen the duration of recumbency, concept of bipolar prosthesis and use of synthetic bone cement was introduced, which reduced the complication of implant failure.

\section{Aims and Objectives}

1. Primary: Functional outcome of cemented bipolar hemiarthroplasty in unstable intertrochanteric fractures in elderly osteoporotic patients.

2. Secondary: To compare the functional outcome of cemented bipolar hemiarthroplasty with DHS in unstable intertrochanteric fractures in elderly osteoporotic patients.

3. Tertiary: To study the complications associated with cemented bipolar hemiarthroplasty in unstable intertrochanteric fractures in elderly osteoporotic patients.

\section{Review of Literature}

Ambrose Pare in 1634 recognized hip fractures.

Philips in 1867 introduced the conservative treatment of hip fractures with longitudinal and lateral traction to eliminate shortening or deformity
Whitman in 1902 advocated careful reduction and holding of fractures in a hip Spica with unpublished result. Watson Jones in 1955 estimated union rate of $40 \%$ using this method Von Langenbeck in 1850 was the first person to use nail for neck of femur.

Smith Peterson used triflanged nail for open reduction and internal fixation of intertrochanteric fractures.

Ender (2) in 1970 reported the use of multiple flexible condylocephalic nails which were introduced for stabilization of intertrochanteric fractures.

Medoff in 1991 described a new device that allowed bidirectional impaction of the fracture. In his study of two unstable intertrochanteric fractureshe noted no cases of implant failure Baumgaertner in 1995 defined the tip apex distance (T.A.D), They defined less than $25 \mathrm{~mm}$ was associated with no incidence of cut out.

Sadowski (6) in 2002 supported the use of intramedullary nail rather than a 95 degree screw plate fixation for sub trochanteric and transverse trochanteric fractures.

\section{Materials and Methods (Methodology)}

- Study Design- Longitudinal study

- Study Settings- Department of Orthopaedics, Government Medical College, Thiruvananthapuram.

- Study Period - Jan 2015 to Sept 2016

- Study Population

\section{Inclusion Criteria}

1. Age of the patient at least $70 \mathrm{yrs}$ and older

2. Femoral intertrochanteric fracture confirmed on antero-posterior and lateral hip radiographs.

3. Should be unstable fracture $\mathrm{AO} /$ Orthopedics Trauma Association. Alphanumeric Classification (A 2.2,A2.3, A3.1,A3.2,A3.3)

4. Patient ambulatory prior to fracture.

\section{Exclusion Criteria}

1. Age less than 70 years 
2. Associated major injuries of lower extremity

3. Any infection around the affected hip.

\section{Surgical Technique}

With patient in lateral position, we used posterolateral lateral modified Gibson's approach. Proximally fibers of gluteus maximus were dissected along skin incision to expose fracture site reaching an end on view of the femoral neck, femur head and the attached neck is extracted through this window. Then we started femoral canal preparation using reamer and serial broach's. In cases highly comminuted greater trochanter two holes on lateral aspect of proximal femur $5 \mathrm{~cm}$ below vastus ridge 2 holes were placed $2 \mathrm{~cm}$ away from each other. A stainless steel wire was passed from outside through one hole in the medullary canal and then taken out from another hole and passed over the greater trochanter in a figure of eight fashion. The implant placement is now started taking into consideration of these facts.

1. Anteversion which is decided by using long axis of the leg as guide as lesser trochanter will be fractured in almost all cases.

Percentage distribution of Sample according to age

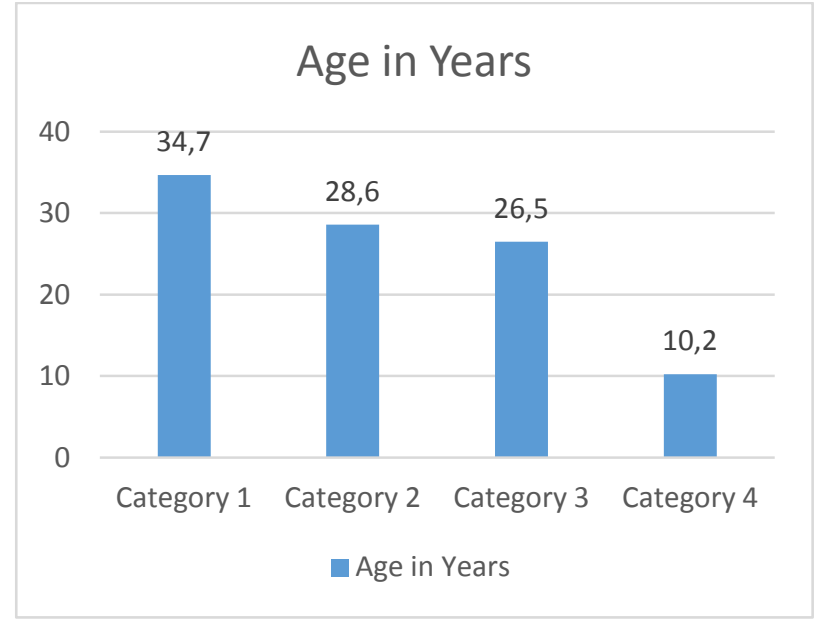

2. Length of the implant to be inserted in the femur is decided by carefully judging soft tissue tension.

3. No excess cement should spread on fracture site as it interferes with union of the fracture

Version, length and so tissue tension of the implant are assessed using trial prosthesis. Second generation cementing technique is used. Once the prosthesis was fixed, the broken trochanter and calcar were again retightened by tensioning the wire cables if needed. And incision is closed in layers with drain.

Post- operative and Rehabilitation Protocol: All patients were treated with Quadriceps strengthening exercises immediately post op and partial weight bearing on $2^{\text {nd }}$ or $3^{\text {rd }}$ post-operative day with the help of walking frame. Thereafter patients started full weight bearing with support of a stick at around 10 to 14 days.

\section{Results and Observation}

There were 49 patients with intertrochanteric fractures of various types in which, 1 patient died and the cause of death was not related to the surgery. The analysis of the patient's data, intra operative data and post-operative outcome is as follows.

\begin{tabular}{|c|c|c|}
\hline Age in years & Frequency & Percent \\
\hline$<75$ & 17 & 34.7 \\
\hline $75-79$ & 14 & 28.6 \\
\hline $80-84$ & 13 & 26.5 \\
\hline$>85$ & 5 & 10.2 \\
\hline Total & 49 & 100 \\
\hline
\end{tabular}

\begin{tabular}{|l|c|c|c|}
\hline \multirow{2}{*}{} & \multirow{2}{*}{$\mathrm{N}$} & \multicolumn{2}{|c|}{ Age } \\
\cline { 3 - 4 } & & Mean & Sd \\
\hline CBA & 24 & 78.7 & 4.6 \\
\hline DHS & 25 & 76.7 & 4.9 \\
\hline
\end{tabular}


Percentage Distribution of the Sample according to Sex

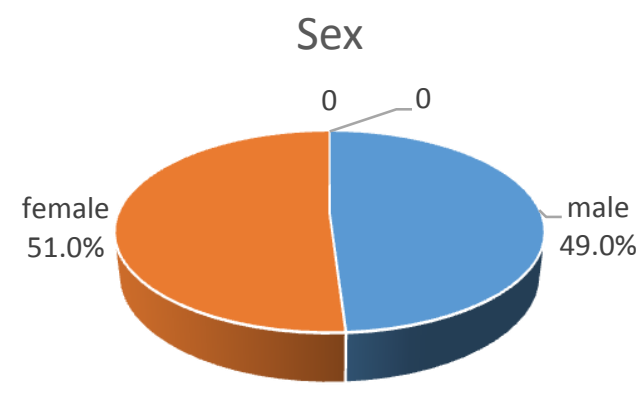

\begin{tabular}{|l|c|c|}
\hline Sex & Frequency & Percent \\
\hline Male & 24 & 49.0 \\
\hline Female & 25 & 51.0 \\
\hline Total & 49 & 100.0 \\
\hline
\end{tabular}

Percentage distribution of the sample according to AO/Classification

\begin{tabular}{|l|c|c|}
\hline Type of factures & Frequency & Percent \\
\hline A2.2 & 11 & 22.4 \\
\hline A2.3 & 14 & 28.6 \\
\hline A3.1 & 15 & 30.6 \\
\hline A3.2 & 1 & 2.0 \\
\hline A3.3 & 8 & 16.3 \\
\hline TOTAL & 49 & 100.0 \\
\hline
\end{tabular}

\section{Functional Outcome}

Out of 49 patients studied we were able to follow up 48 patients and 1 patient died due unrelated medical cause. All patients were subjected to Harris hip score at 2 weeks, 6 weeks, 3 months, 6 months and 1 year follow ups. Patients who underwent cemented bipolar hemiarthroplasty 2 weeks, 6 weeks, 3 months had better Harris hip score than who went DHS and they were statistically significant $(\mathrm{p}<.001)$. Patients who underwent cemented bipolar hemiarthroplasty were more pain free and had greater range of hip movements. They could be mobilized very early compared with the other group and were able to presume their daily activities early than other group. While patients who underwent DHS could not be mobilized early due to pain at the fracture site and both hip and knee joint stiffness. Patients who had low Harris hip score initially continued to have low score finally. In the following months; i.e. $6^{\text {th }}$ month and 1 year both groups had better Harris hip scores. The reason may be that fracture had already united and patients could be mobilized in the DHS group. Harris hip score at final follow up in both groups were comparable and no difference couldn't be found out statistically. Of 49 patients we had $27.1 \%$ patients with excellent Harris hip score, 56.3\% good Harris hip score, $12.5 \%$ patients had fair and $4.2 \%$ had poor scores. Harris hip score had no association with any of the variables used in our study.

Percentage Distribution of the Sample according to Functional Assessment based on Harris Hip Score at 1 year

\begin{tabular}{|l|c|c|c|c|c|c|c|c|c|}
\hline \multirow{2}{*}{ HHS 1 year } & \multicolumn{2}{|c|}{ CBA } & \multicolumn{2}{|c|}{ DHS } & \multicolumn{2}{|c|}{ TOTAL } & \multirow{2}{*}{$\mathrm{X}^{2}$} & $\mathrm{~d}$ df & $\mathrm{p}$ \\
\cline { 2 - 8 } & $\mathrm{N}$ & $\%$ & $\mathrm{~N}$ & $\%$ & $\mathrm{~N}$ & $\%$ & & & \\
\hline Poor & 0 & 0.0 & 2 & 8.3 & 2 & 4.2 & 2.729 & 3 & .435 \\
\hline Fair & 3 & 12.5 & 3 & 12.5 & 6 & 12.5 & & & \\
\hline Good & 13 & 54.2 & 14 & 58.3 & 27 & 56.3 & & & \\
\hline Excellent & 8 & 33.3 & 5 & 20.8 & 13 & 27.1 & & & \\
\hline Total & 24 & 100.0 & 24 & 100.0 & 48 & 100.0 & & & \\
\hline
\end{tabular}


Distribution of Sample Accordingto Post Operative Complications.

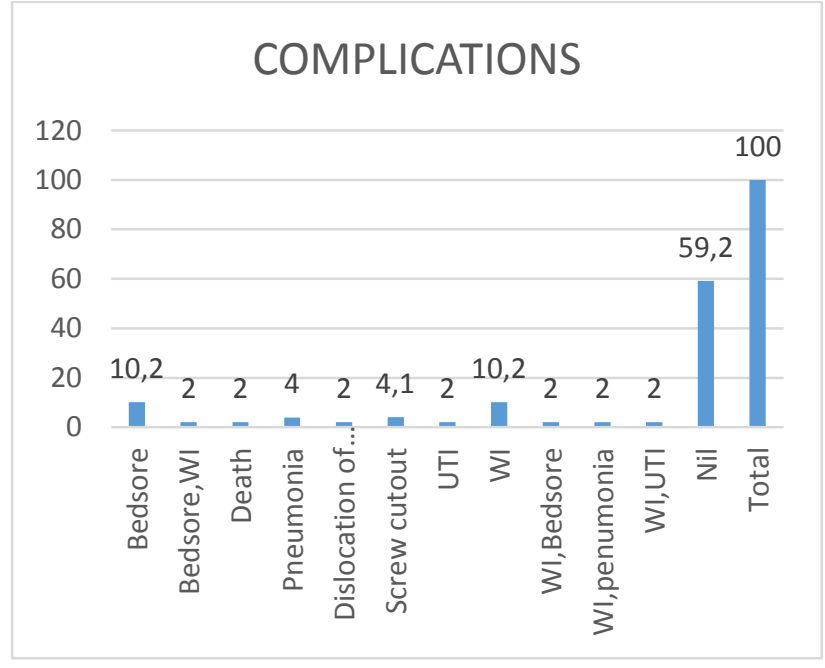

\section{Discussion}

Mortality rate of $34.6 \%$ have been reported for trochanteric fractures treatment with traction only, against $17.5 \%$ for those, treated by operative methods. The incidence of the hip fracture is closely related to age and increases almost exponentially, so that about $90 \%$ of hip fractures occur after age 70 . The cross sectional geometry of the lower limb bones including the femur may play an important role in the difference of the hip fracture risk between women and men.

Indications of bipolar hemiarthroplasty: Grimsrud et al (2005) reviewed 39 consecutive patients with unstable 3 and 4 part intertrochanteric hip fractures who were treated with cemented bipolar hemiarthroplasty. Patients had better prognosis, early full weight bearing, rapid rehabilitation and few complications in the study. In our study we also had similar results. It helped in early mobilization of patient with good and fast improvement in Harris hip score. Complications like pressure sores, aspiration pneumonia are very rare with surgery. Cemented bipolar hemiarthroplasty has given constant good results in terms of early ambulation and good mid-term survival rates, less implant related complications and faster over-all rehabilitation in comminuted unstable intertrochanteric fractures and results are constant as compared to variable results given by DHS. Final outcome in both the groups were more
Distribution of the Sample -

Time of Partial \& Full Wt Bearing

\begin{tabular}{|l|c|c|c|c|c|c|}
\hline & \multicolumn{2}{|c|}{ CBA } & \multicolumn{2}{c|}{ DHS } & $\mathrm{t}$ & $\mathrm{p}$ \\
\cline { 2 - 7 } & Mean & sd & Mean & sd & & \\
\hline $\begin{array}{l}\text { Time of partial } \\
\text { weight } \\
\text { bearing(days) }\end{array}$ & 3.9 & 2.2 & 27.7 & 2.6 & 34.706 & $<0.001$ \\
\hline $\begin{array}{l}\text { Time of full } \\
\text { weight bearing } \\
\text { (days) }\end{array}$ & 13.2 & 2.6 & 176.2 & 4.1 & 1565.120 & $<0.001$ \\
\hline
\end{tabular}

or less comparable except for the fact that there was early ambulation in hemiarthroplasty group. Certain studies have also shown that there are higher rates of dislocation with posterior approach when compared with lateral trans trochanteric and antero-lateral approach. Hence we used lateraltrans trochanteric approach for our patients. Stainless steel wire wiring technique for fixation of greater trochanter as shown in case series by Zhang Q et al. We had used this technique for stabilization for highly comminuted fractures in our study. In our study we has encountered no delayed or non-union of greater trochanter.

Functional results: Bipolar prosthetic replacement can be recommended as the preferred treatment in selected cases of unstable comminuted intertrochanteric fractures in the elderly. In such cases augmentation with cement fixation is a must. Rodop et al. in a study of primary bipolar hemi prosthesis for unstable intertrochanteric fractures in 37 elderly patients obtained 17 excellent (45\%) and 14 good (37\%) results after 12 months according to the Harris hip scoring system. A total of 18 out of 23 patients in our study had a good to excellent result $(71 \%)$. The Harris hip score at 2 weeks, 6 weeks and 3 months post operatively was significantly higher in patients who underwent bipolar arthroplasty (76.15 \pm 6.11$)$ (Range 68-85) compared to those 
in the internal fixation group (64.89 \pm 5.66$)$ (range 61-75) which is statistically significant ( $\mathrm{p}<0.001)$. Our results were comparable to study by Sancheti et al and Elmorsy et al which has shown that primary bipolar hemiarthroplasty in comminuted extra capsular neck femur fracture which provided early mobilization, stable and pain free hip with early rehabilitation and return to daily routine life with less reoperation is fairly economical especially for developing countries. Early infections can be either superficial or deep. Complications in our study included superficial infections only and no deep infections. All superficial infections healed with prompt treatment. The findings in present study suggests that primary cemented bipolar hemiarthroplasty is a good choice for unstable intertrochanteric fractures in the elderly and saves time as well as cost, has no significant complications and provides sustainable functional outcomes.

\section{Limitations of the Study}

The limitation of present study is the short duration of follow up.

\section{Conclusion}

Hemiarthroplasty using bipolar prosthesis for the unstable intertrochanteric fractures of the femur in elderly has good clinical result in terms of early post-operative ambulation. Primary hemiarthroplasty thus provide a stable, pain-free and mobile joint with acceptable complication rate as seen in our study however a large prospective randomized study comparing the use of intramedullary devices against primary hemiarthroplasty for unstable osteoporotic fractures will be needed. Final outcome depends on the general condition and post-operative rehabilitation of the patient. Patient selection is very important as we are directing this to the elderly people with osteoporotic bone and have unstable intertrochanteric fractures. This also improves their quality of life and the ability to go back to their original functional status much earlier than dynamic hip screw hence bipolar hemiarthroplasty should be considered as one of the modalities of the treatment of unstable osteoporotic inter trochanteric fractures in elderly.

\section{Bibliography}

1. Bucholz RW, Heckman JD, Koval RJ, Zukerman, Rockwood and Grends fracture in adults, 6 th edition. Philaldephia, Lippincott Williams \& Wilkins; 2005

2. Chapman MW, Bowman We, The use of enders pins in extra capsular fractures in hip JBJS Am 1981;63:14-28

3. Sadowski $\mathrm{C}$,Lubbke $\mathrm{M}$.treatment of reverse oblique and transverse fractures of intertrochantericfractures with the use of intramedullary nail or a 95 degree plate JBJS Am2002:84-A: 372-381

4. Alffram PA, An epidemiological study of cervical and trochanteric fractures of femur in an urban population: Analysis of 1664 cases with special reference to etiological factors. Acta Ortho Scand 1964;65-9-109

5. Cumming S, Black D, Nevitt M. Bone density at various sites for prediction of hip fractures. Lancet 1993; 341: 72-75

6. Koval KJ, Zuckerman JD ,Hip fractures ; a practical guide to management .New York; Springer- Verlag:2000

7. Horn SR, Wang M, the mechanism, traumatic anatomy and treatment of intertrochanteric fractures of femur. $\mathrm{Br}$ journal of surgery.1964;51:234-245.

8. Boyd HB, Griffin LL Classification and treatment of intertrochanteric fractures.

Arch surg 1949; 58:853-866

9. Muller ME, Nazarian $S$, Koch $P$, Schatzker J. the comprehensive classification of fractures of long bones. Berlin; springer Verlag 1990.

10. Demon JH, Hughson JC.1967. Unstable intertrochanteric fractures of hip , J Bone Joint Surg, 49-A: 440-50

11. Ecker Malcom L. et.al. 1975, treatment of trochanteric hip fractures using 
compression screw, J Bone Joint Surg 57

A: 23-27.

12. Bannister G.C, Gibbson A.G.F 1983, Jewett Nail Plate or AO dynamic Hip S screw for trochanteric fractures. A randomized prospective controlled trial. $\mathrm{J}$ Bone Joint Surg( Br) 65B;218. 